\title{
Modelo pedagógico para la interactividad de las ciencias técnicas Aproximación conceptual
}

\section{Model teaching for the interactivity of the sciences technical Approach conceptual}

\author{
págs. 106-113 \\ Grupo de investigación: Grupo Laboratorio de Pedagogía y Didácticas de las Ciencias Experimentales y de \\ las matemáticas - LabEX \\ Línea de investigación: Problemas teoricos de la Pedagogía en las Ciencias Técnicas - Ingeniería Química \\ Claudio Raúl Bernal Bustos• Miguel Alfonso Morales Granados••
}

\section{Recibido: agosto 01 de 2016 Aceptado: noviembre 25 de 2016}

\section{RESUMEN}

La solución a un problema no es necesariamente la respuesta favorable, es el soporte, el método de conocimiento con el cual ocurre la respuesta. La presente investigación presente concilia un método de conocimiento que florezca al integrar diversos métodos de conocimientos provenientes de la integración de ciencias diversas alrededor de la solución de un problema común y en la perspectiva de brindar soluciones técnicas y tecnológicas. El modelo pedagógico, que permite comprender el fenómeno educativo que suscita de la integración-articulación de las diversas ciencias y sus métodos de conocimiento, debe reconstruir la enseñanza de las ciencias técnicas a partir de un syllabus superior, y a partir de este, dar forma a la linealidad del syllabus académico.

Palabras clave: Pedagogía, enseñanza técnica, formación profesional, ingeniería química, syllabus.

\begin{abstract}
The solution to a problem not is necessarily the answer favorable, is the support, the method of knowledge with which occurs the response. This research reconciles a method of knowledge that thrives by integrating various methods of knowledge from the integration of Sciences around a common problem solving and in the perspective of providing technical and technological solutions. The pedagogical model, which allows to understand the educational phenomenon arising from the integracion-articulacion of the various sciences and their methods of knowledge, must rebuild the teaching of technical sciences from a superior syllabus, and from this, to give shape to the linearity of the academic syllabus.
\end{abstract}

Key word: Pedagogy, technical education, vocational training, chemical engineering, syllabus.

- Químico. Docente Investigador. Fundación Universidad de América. Correo: claudio.bernal@investigadores.uamerica.ed.co

•• Ingeniero Mecánico. Docente investigador. Fundación Universidad de América. Correo: (¿?) 
LÍNEA DE INVESTIGACIÓN: PROBLEMAS TEÓRICOS DE LA PEDAGOGÍA EN LAS CIENCIAS TÉCNICAS

\section{INTRODUCCIÓN}

Los procesos de Investigación, Desarrollo e Innovación, acrónimo I+D+i, cambian con los fenómenos de globalización, requieren éstos la integración de grandes conjuntos de actividades, subsistemas y equipos de trabajo de variada formación.

La Fundación Universidad de América, y en sus labores misionales de extensión se propone articular los resultados de investigación, las evidencias científicas y el desarrollo de tecnologías con su entorno natural; la sociedad. Dicha articulación focaliza, en una de sus perspectivas misionales, el sector agro-productivo colombiano, principalmente para contribuir en su transformación y competitividad.

El Sistema de I+D+i Institucional, consolidado a través de los grupos de Investigación, plantea que para articular la sociedad con los resultados que provee el Sistema, requiere dinamizar las labores académicas habituales, superando el inmediatismo del Syllabus el cual se encuentra centrado en el cumplimiento de contenidos y saberes de un área de conocimiento particular. Reiteramos que el enfoque direccional del syllabus debe ser así, pero, en el contexto de áreas de conocimiento como es el caso de la ciencias técnicas; caso de las ingenierías y cuyo objeto de conocimiento es la producción de valores de consumo, perdonen el sesgo, requiere de un "momentun" que transgreda la linealidad de los contenidos hacia la visualización de la integración con nuevas áreas de conocimiento, con las nuevas dinámicas de globalidad. Trapéznikov, (1973) ya lo manifestaba, en su momento, "que la conquista del cosmos, el problema ecológico, la mecanización y automatización de la producción y otros problemas globales no pueden ser estudiados con los mismos medios de una teoría o de una disciplina científica, e incluso, ciertos problemas mucho más específicos busquen el apoyo de las teorías existentes de dominios inimaginables".

Para ejemplificar esta situación no debe ser extraño que un trabajo de grado localizado en las bibliotecas institucionales compile un número igual o mayor a 300 hojas, donde su contenido está conformado por muchas tareas y actividades que no hicieron parte "explícita" del syllabus, además, ha llevado un promedio de 2 años su realización y, por lo general, es documentación "muerta".

Planteamos, entonces, un primer ejercicio dirigido a integrar contenidos y saberes diferentes en dos momentums, el de los contenidos (Syllabus) y el objeto de estudio (problema científico), existiendo una brecha entre estos, como lo señala Bernal, C. (2014); brecha de conocimiento entre lo que el estudiante puede hacer y lo que el estudiante debe hacer, así como entre lo que el estudiante sabe y lo que el estudiante debe saber del objeto de estudio durante la actividad de estudio (Davidov, 1988).

Se pretende integrar algunos de las partes de la estructura del Syllabus de la Ingeniería Industrial, Mecánica, Química, Petróleos, Economía, entre otras como la de Ambiente y Hábitat (Bernal, C. et al., 2015); partes que en algún momento podrían hacer parte de un Syllabus Superior y que darían fundamento académico a la solución de un problema científico común. Dicha integración, o Syllabus Superior, suscita en el interés de fortalecer uno de los elementos que le son inherentes al pensamiento científico-técnico; la "construcción", alrededor de la creación y manufactura de prototipos de procesos y de productos, como respuesta tangible al problema.

Para la elaboración de un syllabus superior, como elemento visible de la integración de saberes; con sus métodos, alrededor de un problema científico común y cuyos resultados tangibles son los 
constructos tecnológicos, debe recurrir y en un primer comienzo, a dar fundamento a la aparición de un fenómeno educativo que requiere comprenderse. La ciencia pedagógica, cuyo objeto de conocimiento es la comprensión de los fenómenos de la educación, aparece como principal herramienta conceptual para tales fines.

\section{MÉTODO}

La integración de conocimientos se ha convertido en el fenómeno educativo de estudio de los Grupos de Investigación: LabEX - Laboratorio de Pedagogía y Didácticas de las Ciencias Experimentales y de las Matemáticas y Simulación e Instrumentación para la Docencia, advirtiendo que no es un asunto exclusivamente técnico y de las disciplinas, que obedece a un asunto epistemológico, así como a leyes de la educación, a la sociología y ética (Bernal, C. 2014, pág. 133).

Este mismo autor señala que para realizar integraciones de conocimientos y métodos de campos distintos de saber, es necesario y de manera inicial la comprensión de la articulación entre estadíos al interior del mismo saber, por ejemplo, del paso de un estadío a otro: del ciclo básico hacia el profesional en la formación de ingenieros químicos, siendo una transición entre el conocimiento de las ciencias básicas hacia las aplicadas y propias del campo acotado de las ciencias técnicas. Este autor alerta cuatro situaciones problema encontradas en sus investigaciones: en primer lugar, lo referido al cuerpo de conocimientos o conjunto de conceptos científicos; asimilados como cuerpos ya acabados, en segundo lugar, las explicaciones del estudio de los fenómenos sobre la base de la experiencia e intuiciones, tercero, la dificultad al tomar decisiones experimentales y cuarto, la posibilidad de la negociación conceptual entre los mismos estudiantes, entre estudiantes-docentes y estudiantes-sociedad.

Consecuente con lo anterior, la universidad de América declara que en su enfoque pedagógico constructivista se busca que los aspectos cognitivos, sociales y afectivos del estudiante sean una construcción propia que se va produciendo día a día como resultado de la interacción con el medio, con los docentes y con sus mismos compañeros de estudio, que el conocimiento es producto de una construcción social y de la misma manera el sujeto se constituye en la interacción social y cultural. (America, 2010)

Recordemos, que la relación estudiante (profesional)-sociedad, es la relación que define el paso por un proceso educativo formal profesional, en todo caso, y de acuerdo con las ideas filosóficas modernas, el hombre que asimila los conocimientos existentes ya no trata con la realidad inmediatamente circundante, por cuanto el "objeto de conocimiento esta mediatizado por la ciencia como formación social, por su historia y por la experiencia... en ese objeto se han separado determinados aspectos, dados al individuo que ingresa en la ciencia, en forma de contenido generalizado, abstracto, de su pensamiento. (M. Mamardashvili. Forma y contenido del pensamiento. Moscú, 1968, pág. 21).

Lo anterior plantea una situación problema al momento de comprender el fenómeno de estudio, en relación a la integración de áreas de conocimiento distintas, cada una con métodos de conocimiento particular y alrededor de brindar solución a un problema común, para lo cual, la situación de cambio de estadíos; con sus cuatro elementos problema, y la mediatización por la ciencia del objeto de conocimiento, son las principales barreras que se deben superar. No obstante, en la aproximación conceptual presente, la situación arriba señalada es analizada fuera del contexto académico, se toma como elemento discursivo el trabajo al interior de un proceso de investigación científica; cuyo inte- 
LÍNEA DE INVESTIGACIÓN: PROBLEMAS TEÓRICOS DE LA PEDAGOGÍA EN LAS CIENCIAS TÉCNICAS

rés redunda en favor del desarrollo de tecnologías involucrando aportes concretos de la Ingeniería industrial (modelamiento de procesos), Ingeniería mecánica (diseño y desarrollo de prototipos y su control), de las ciencias economías (estudios de factibilidad financiera e impactos sociales), entre otras disciplinas aportantes, dando lugar además que los estudiantes puedan fortalecer competencias propias del modelo constructivista tales como (Tuning, 2010): a) Capacidad de buscar, procesar y analizar información de fuentes diversas; b) Capacidad de trabajo en equipo; c) Capacidad de aplicar conocimientos en la práctica; Capacidad de investigación, Capacidad de formular y gestionar proyectos.

El objetivo e interés de este avance de investigación, principalmente suscita alrededor de la formación de Ingenieros Químicos, pero, cuando éstos se encuentran en la última etapa de su formación profesional, realizando su trabajo de grado en la modalidad de investigación y al interior de grupos de investigación. La experiencia a estudiar se encuentra acotada en la definición o desarrollo de una tecnología para la producción optimizada de almidones funcionalizados a grados industriales.

Las acciones científicas iniciadas y planteadas toman su fundamento en la experiencia tácita al argumentar lo siguiente: los resultados de investigación; básica o aplicada y según la tipología de Colciencias, deberían ser llevados a proyectos de desarrollo tecnológico y de aquí a la industria, sin embargo, es claro delimitar la función del "escalamiento de productos y de procesos"; máximo, de la acción académica: prototipo, a escala aumentada, sin fines comerciales, siendo el único interés la validación de las teorías (Tipologías de Colciencias).

La Investigación da inicio desde los diseños y desarrollos a escala de laboratorio, cuyo objetivo, y sobre el interés de la tecnología, centró la atención en establecer un "patrón de uso" de los almidones; materia prima principal de la investigación. Con el objeto de obtener un producto potencialmente comercial en el futuro, se inicia las etapas de ingenierización: Laboratorio $\rightarrow$ banco $\rightarrow$ a planta piloto $\rightarrow$ desarrollo industrial, lo que conlleva a establecer situaciones diferenciadas de conocimientos, de métodos de conocimientos e integración a métodos de conocimiento universales.

El fenómeno educativo se origina cuando se deben establecer las fronteras de los conocimientos aportantes que llevarían a la definición del syllabus superior. No obstante, en este artículo, no se alcanza a diferenciar el fenómeno de estudio, dado a que de manera inicial; desde el syllabus de la ingeniería química, se inician los desarrollos a escala de laboratorio realizando modificaciones físicas, químicas y biotecnológicas sobre el ingrediente natural; previo fases de extracción $\rightarrow$ purificación $\rightarrow$ caracterización $\rightarrow$ modificación, para finalizar en la $\rightarrow$ certificación del ingrediente como una materia prima. En todo caso, en este estadío aún no se integran los diferentes conocimientos.

La fase de extracción, por citar una situación técnica, involucra revisar conocimientos desde la química que conlleven a determinar múltiples referentes, por ejemplo, la comprensión de la cinética del fenómeno de extracción sólido-líquido; previa comprensión de la matriz biológica y química donde se encuentra alojado, además del ingrediente de interés, otras sustancias tal es el caso de los "cuerpos proteícos y grasos" que hay que "vencer", sin desconocer la importancia de los minerales; que en conjunto se encuentran almacenados como reservas en las plantas y principalmente en semillas.

El desarrollo de, prácticamente, todas las operaciones de un laboratorio químico va unido al manejo de materiales en porciones de considerable peso y volumen. En la etapa de desarrollo, a escala de laboratorio, según Bebedetti-Pichler; en estas pequeñas cantidades hay todavía un número 
suficiente de moléculas presentes para poder establecer una relación reproducible, y que sólo en muestras tan reducidas como $10^{-16}$ gramos o menores, por ejemplo; el equilibrio químico, está sujeto a cambios, al variar la masa absoluta del sistema. Así, la química permanece inalterable en la escala micro, y la única cuestión es que las técnicas de manipulación sean apropiadas al momento de aumentar la "escala". Lo anterior, teniendo en cuenta, por ejemplo, que cuando están implicadas consideraciones químicas, el estudio se aplica a todos los momentos; escala micro y macro, y se evita la repetición. Por otra parte, se indican también ciertas propiedades físicas que presentan una diferencia de relativa importancia al cambiar la escala. Por su puesto, éstas se tratan en estudios de microanálisis y en el que el aspecto cuantitativo es fundamental, pero su impacto es totalmente general. Así, la tensión superficial tiene una importancia extraordinaria en el manejo de los líquidos; y una reducción de escala aumenta la importancia relativa de superficie por aumentar la relación superficie-volumen.

De las problemáticas que suscitan en el fenómeno de extracción de almidón -ejercicio analizado a partir de la extracción de granos-semillas de quinua- tiene que ver con el fenómeno de la filtración; momento seguido a la relación soluto-líquido y la capacidad de la difusividad térmica del líquido por los intersticios del sólido y "sobrellevando" la presencia de sustancias más afines al propio ingrediente de interés. Esto no es más que comprender el fenómeno que rige la transferencia de masa durante la lixiviación del almidón en agua mediante la cinética del proceso y donde los parámetros de operación del sistema, como: tamaño de partículas, temperatura, presión, velocidad de agitación y tiempo de extracción están establecidos. Sin embargo, la determinación de los parámetros cinéticos de este proceso de transferencia de masa no necesariamente está informado en la literatura científica.

En este artículo no podemos informar, aún, el modelo seleccionado para describir la cinética de lixiviación del almidón de quinua en agua, no obstante sabemos que la constante cinética de la velocidad de extracción depende de la naturaleza del sistema sólido-líquido y de la temperatura (Wallas SM. 1979). La experimentación nos llevará a obtener la constante cinética, lo que permitirá calcular el valor de la energía de activación, indicando que el proceso de transferencia de masa es difusivo, ya que según Habashi (1980) cuando este valor es inferior a nKJ/mol el proceso de lixiviación está controlado por la difusión, es decir, por vía física.

\section{RESULTADOS}

El cambio significativo supone la integración de los diferentes campos acotados de conocimiento (Ingenierías química, mecánica, etc.); ahora conformadas; cada una de éstas, en unidades básicas: Ingeniería Química - Unidad Básica 1, Ingeniería Mecánica - Unidad Básica 2, Ingeniería Industrial Unidad Básica 3, etc. Realizada esta diferenciación, por Unidades, surgen las siguientes preguntas: ¿Cuándo consideramos que hay dos unidades básicas distintas?, o ¿qué tiene que cambiar en una unidad básica para que consideremos un cambio como significativo aportante al problema común Estructura 2?, Podemos decir que dos unidades básicas no son iguales si tienen, por lo menos, un elemento distinto. Será significativo, en primer grado, todo cambio que implique una variación de alguno de los elementos constitutivos de las Unidades Básicas - UB, principal razón de Syllabus Superior.

Los elementos comunes y constitutivos que conforman las Unidades Básicas, son: la base teórica, la ontología de la base teórica, su aplicación o campo de impacto, los procedimientos científicos, los instrumentos y técnicas. Para responder a las preguntas, es necesario, en primer lugar, entender 
que se corresponde a una especificación elemental en el sentido de que el cambio de uno de los elementos es necesario y a la vez es suficiente para que haya dos unidades básicas distintas.

Elementos constitutivos de la Unidad Básica (Stany, 1990): (1) Base teórica. Es el primer elemento en una UB, se encuentra constituido por el conjunto de teorías, reglas, principios y leyes y, en general, por todo el conjunto de conocimiento sistematizado que se tiene sobre un campo acotado de fenómenos; (2) Ontología de la base teórica, son los elementos sobre los cuales se teoriza y a partir de los cuales se formulan las leyes; (3) Aplicación, conjunto de fenómenos que son explicados por el cuerpo teórico. A veces puede constatarse una relación inversa entre la extensión del campo de aplicación y el poder explicativo de la teoría o conjunto de teorías; sin embargo, no puede afirmarse que siempre sea así y, por tanto, esta afirmación no puede tomarse como principio meta teórico para servir de guía en la investigación científica; (4) Procedimiento cientifico, siendo todos aquellos principios que actúan como guías y que subyacen a todo el quehacer científico, marcando cómo ha de realizarse la investigación; (5) Instrumentos y técnicas, interacción entre la disponibilidad de éstos y el poder explicativo de las teorías. Sin embargo, no siempre la interacción es con las teorías del campo científico en el que se quieren utilizar los instrumentos, sino que puede darse el caso de que un campo científico progrese debido a la utilización de unos instrumentos que han podido construirse gracias al desarrollo teórico de otro campo científico distinto al primero. Adicional a estos elementos constitutivos, se plantea un elemento nuevo; a o planteado por Stany, (1990), tiene que ver con el (6) Modelo pedagógico. Congruentes con las directrices de la Fundación Universidad de América se promueve el modelo Constructivista de Vygotsky y se favorece estrategias formativas como el aprendizaje colaborativo por la interdisciplinariedad del proyecto donde estudiantes de diferentes disciplinas deben interactuar permanentemente transfiriéndose información con el fin de lograr un objetivo común como una solución a una problemática planteada, también se favorece el aprendizaje basado en problemas en donde el estudiante investigador se enfrenta a situaciones específicas dando lugar a que el entendimiento de una situación de su quehacer profesional en la interacción con el medio ambiente le estimula su proceso cognitivo y el espíritu investigativo. El Gráfico 1 deja ver, de forma esquemática; representadas en colores las UB y sus elementos, como podrían interactuar-articularse, para conformar un Syllabus Superior y éste servir de argumentobase del problema científico. Se deja ver, además, el surgimiento del fenómeno educativo.

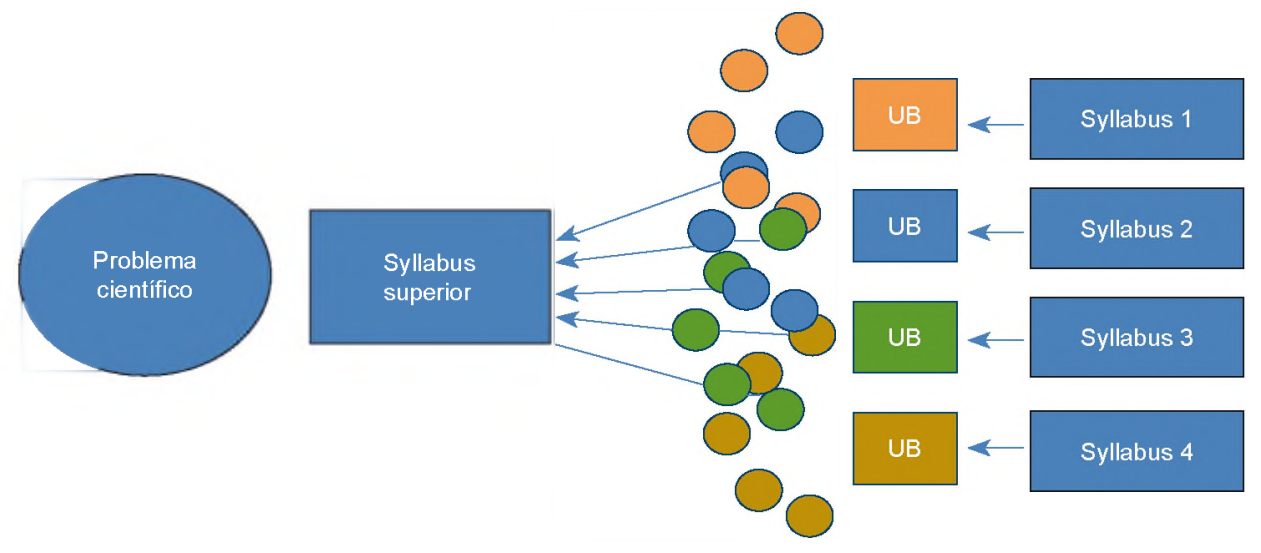

Gráfico 1. Representación esquemática del surgimiento del Syllabus Superior y su articulación al problema científico.

Fuente: Autores 
La representación gráfica no precisa los subelementos de cada elemento, por ejemplo, en el caso de la base teórica - syllabus 1; puntos naranja, ésta obedece a diversas estructuras, tal y como lo expone Stany (1990), existiendo otros elementos definidos por este autor como estructuras de dominio ordenadas, estructuras de conjunto compacto, de correlaciones, de concepto explicativo preferente. Según el gráfico, algunos puntos; subelementos (colores), interaccionan entre sí, siendo esta interacción los subelementos que se dan fundamento al syllabus superior. Así mismo, de estos elementos, Stany (1990) en su modelo de cambio científico plantea la existencia de algunas configuraciones teóricas que harían parte del cuerpo teórico. La autora denomina teorías de apoyo y teorías colaterales. El modelo pedagógico, para el caso de dar argumento al syllabus superior planteado, considera importante tomar cada uno de los elementos como constitutivos en la propuesta de cambio científico (Stany, 1990), pero, debe estudiar el argumento de las técnicas y métodos de enseñanza; la teoría didáctica, en la situación particular, por un lado, la comprensión de la situación que suscita al integrar las diferentes ciencias y sus métodos y, por el otro, en la definición de todos y cada uno de los procedimientos científico alrededor del problema común.

\section{CONCLUSIONES}

En el contexto anteriormente planteado, la investigación ha centrado su atención en definir los diferentes elementos constitutivos que darán forma al syllabus superior o sus contenidos procedentes de Unidades distintas; junto a sus métodos, pero, estos contenidos; por si solos no representan la comprensión del fenómeno educativo, debe acudir a las técnicas y métodos de su enseñanza, es decir, a una didáctica del syllabus superior.

\section{AGRADECIMIENTOS}

Sistema de Bibliotecas"Baldomero Sanín Cano", Colecciones Digitales (Ebsco, ScienceDirect, Springer Link, IOPscience) y Software Celsius Network - Fundación Universidad de América. 2016.

\section{REFERENCIAS}

América, F.U. (2001). PEI (Proyecto Educativo Institucional). Bogotá.

Bernal, C. y Espinoza, H. (1998). Exploración psicopedagógica acerca de la actividad intelectual que se promueve en los estudiantes durante la experimentación científica. Fase I. Revista de Investigación, Fundación Universidad de América, V1 pp.133-150.

Bernal, C. (2014). De las ciencias básicas hacia las ciencias técnicas durante la formación profesional. Experiencias en Ingeniería Química. Tomo 17, Capítulo 11. Colección Pedagogía Ibero Americana; Escenarios de la Educación, la Enseñanza y el Aprendizaje. ISBN 978-95858674-4-4, Primera Edición, Noviembre del 2014. Editorial REDIPE.

Bernal, C. (2014). Construcciones teóricas sobre las acciones metodológicas preliminares en laboratorio durante la formación de ingenieros químicos. Revista Educación e Ingeniería - ACOFI, Vol.9 No. 18, pp. 206-215. 
LÍNEA DE INVESTIGACIÓN: PROBLEMAS TEÓRICOS DE LA PEDAGOGÍA EN LAS CIENCIAS TÉCNICAS

Bernal, C. (2014). Frontera entre las ciencias biomoleculares y la ingeniería bioquímica - aproximación Dialéctica. Encuentro Internacional de Educación e Ingeniería, ACOFI 2014; Escenarios en la enseñanza de la Ingeniería - Texto Original en CD; anexo memorias.

Davidov, V. (1988). La modelación como medio del pensamiento científico. La enseñanza y el desarrollo psíquico, pp132. Moscú.

Habashi F. Principles of Extractive Metallurgy, Volumen 1. General Principles. New York-LondonParín: Gordon \& Breach Science; 1980.

Stany, Anna. (1990). Modelos de cambio científico. Editorial Crítica, S.A., Aragó, Barcelona.

Trapéznikov (1973). La ciencia filosófica marxista-leninista y la contemporaneidad - Voprost filosofil, M., No.8, p. 18.

Tuning. (03 de 11 de 2010). Proyecto Tuning America Latina. Obtenido de Tuning América Latina: http://www.tuningal.org/es/competencias/geología.

Wallas SM. Cinética de las reacciones químicas. La Habana: Editorial Pueblo y Educación; 1979. 